\title{
Influence of aging paths on the thermal runaway features of lithium-ion batteries in accelerating rate calorimetry tests
}

\author{
Xuning Feng ${ }^{1}$, Dongsheng Ren ${ }^{2}$, Shunchao Zhang, ${ }^{2}$, Xiangming He ${ }^{1, *}$, Li Wang $^{1}$, Minggao Ouyang ${ }^{2, * *}$ \\ ${ }^{1}$ Institute of Nuclear and New Energy Technology, Tsinghua University, Beijing 100084, P R China; \\ ${ }^{2}$ State Key Laboratory of Automotive Safety and Energy, Tsinghua University, Beijing 100084, P R \\ China \\ "E-mail: hexm@mail.tsinghua.edu.cn; ouymg@ mail.tsinghua.edu.cn
}

doi: $10.20964 / 2019.01 .14$

Received: 2 September 2018 / Accepted: 12 October 2018 / Published: 30 November 2018

\begin{abstract}
Aging is inevitable during the use of lithium-ion batteries. However, the influence of aging paths on the safety of the lithium-ion batteries remains unclear, leaving uncertainties about safe operation throughout their full life cycle. This paper studies the influence of aging paths on the thermal runaway features in lithium-ion batteries using ARC. Characteristic temperatures are defined to quantify the thermal stability of lithium-ion batteries. Two kinds of aging tests are designed, high-temperature storage and lowtemperature cycling. The effects of aging on the change in the characteristic temperatures have been investigated, providing a quantified analysis of the evolution of battery safety performance during aging. The thermal stability of the cells after low-temperature cycling is worse than that of the fresh cells and less than that of the cells that are treated by high-temperature exposure. Although the capacity retention rates of the cells aged by high-temperature exposure and low-temperature cycling can be similar, their thermal stabilities are quite different. The consumption of active lithium at the anode surface to generate a new SEI layer will result in a better thermal stability in the cells that are treated by high-temperature exposure. However, if there is lithium deposition on the surface of the anode, the thermal stability of the lithium-ion battery will become worse. The quantitative discussions and conclusions of this paper can provide guidance on evaluating the safety throughout the full life cycle.
\end{abstract}

Keywords: lithium-ion battery; energy storage; battery safety; thermal runaway; aging

\section{FULL TEXT}

(C) 2019 The Authors. Published by ESG (www.electrochemsci.org). This article is an open access article distributed under the terms and conditions of the Creative Commons Attribution license (http://creativecommons.org/licenses/by/4.0/). 